\title{
Proprotein convertase expression and localization in epidermis: evidence for multiple roles and substrates
}

Pearton DJ, Nirunsuksiri W, Rehemtulla A, Lewis SP, Presland RB, Dale BA. Proprotein convertase expression and localization in epidermis: evidence for multiple roles and substrates.

Exp Dermatol 2001: 10: 193-203. (C) Munksgaard, 2001

Abstract: Specific proteolysis plays an important role in the terminal differentiation of keratinocytes in the epidermis and several types of proteases have been implicated in this process. The proprotein convertases (PCs) are a family of $\mathrm{Ca}^{2+}$-dependent serine proteases involved in processing and activation of several types of substrates. In this study we examined the expression and some potential substrates of PCs in epidermis. Four PCs are expressed in epidermis: furin, PACE4, PC5/6 and PC7/8. Furin is detected in two forms, either with or without the transmembrane domain, suggesting occurrence of post-translational cleavage to produce a soluble enzyme. In addition the furin active site has differential accessibility in the granular layer of the epidermis relative to the basal layer, whereas antibodies to the transmembrane domain stain both layers. These findings suggest that furin has access to different types of substrates in granular cells as opposed to basal cells. PC7/8, in contrast, is detected throughout the epidermis with antibodies to both the transmembrane and active site and no soluble form observed. A peptide PC inhibitor (dec-RVKR-CMK) inhibits cleavage of Notch-1, a receptor important in cell fate determination that is found throughout the epidermis. Profilaggrin, found in the granular layer, is specifically cleaved by furin and PACE4 in vitro at a site between the amino terminus and the first filaggrin repeat. This work suggests that the PCs play multiple roles during epidermal differentiation.

\section{David J. Pearton', Wilas Nirunsuksiri, ${ }^{1,3}$, Alnawaz Rehemtulla ${ }^{2}$, S. Patrick Lewis ${ }^{1}$, Richard B. Presland ${ }^{1}$ and Beverly A. Dale ${ }^{1}$}

${ }^{1}$ Departments of Oral Biology, Periodontics, Biochemistry, Medicine/Dermatology, University of Washington, Seattle, WA 98195; '2Department of Radiation Oncology, University of Michigan, Ann Arbor, MI; ${ }^{3}$ Present address Dow AgroSciences LLC, Indianapolis, IN

\section{Introduction}

The sequential differentiation of keratinocytes in the epidermis is a complex, highly regulated series of events that results in the formation of the anucleate cornified cells of the stratum corneum from the relatively undifferentiated cells of the basal layer. This differentiation process is calcium dependent and requires extensive and tightly controlled changes in both protein expression and enzymatic activities resulting in characteristic morphological changes of the cells. Proteolytic processing is an important mechanism for many of these events, including signaling, proprotein activation, structural remodeling and desquamation. A number of different proteases have been iden- tified that play a role in epidermal differentiation. These include calpain I (1), cathepsins C $(2,3)$, B, $\mathrm{H}$ and $\mathrm{L}$ (4), profilaggrin endopeptidase (PEP1) (5), the stratum corneum chymotryptic-like protease (SCCP) and the stratum corneum thiol protease (SCTP) (6). The importance of proteases in the epidermis is highlighted by the finding that null mutations of cathepsin $\mathrm{C}$ result in palmoplantar keratoderma disorders, specifically Haim-Munk and Papillon-Lefevre syndromes $(2,7,8)$.

A protease family that has been implicated in processing and differentiation in a number of tissues is the Proprotein Convertase (PC) family. This is a family of $\mathrm{Ca}^{2+}$-dependent serine proteases related to bacterial subtilisin (for reviews see 9-12). To date 7 family members have been iden- 
tified; furin (also known as PACE), PC2, PC1/3, PACE4, PC4, PC5/6 and PC7/8/LPC. Of these furin, PACE4, PC5/6 and PC7/8 are widely expressed whereas $\mathrm{PC} 2$ and $\mathrm{PC} 1 / 3$ are limited to neuro-endocrine tissues and PC4 is restricted to the testis, suggesting both generalized and tissue-specific patterns of expression and function. The PC enzymes recognize basic motifs, cleaving after paired basic residues (PC2 and $\mathrm{PC} 1 / 3$ ) or after a canonical $\mathrm{RX}(\mathrm{R} / \mathrm{K}) \mathrm{R}$ motif (furin and PACE4); in some substrates a P6 Arg can substitute for the Arg at the P4 position (13). Furin has been shown to process a wide variety of substrates including receptors, growth factors, hormones, plasma proteins, matrix metalloproteinases and extracellular matrix components. In addition PC family members are also involved in the processing of exogenous proteins such as some viral envelope glycoproteins and bacterial endotoxins (12).

Several proteins relevant to keratinocyte development have been shown to be substrates for PC processing or contain potential PC cleavage sites. These include receptors such as Notch-1 $(14,15)$, matrix components such as collagen XVII (BP180) (16), matrix metalloproteinases, desmosomal components such as desmoplakin-1 and desmoglein, integrins including integrins $\alpha 3$ and $\alpha 6$ (17) and structural proteins such as profilaggrin.

Although PCs have diverse substrates and play a role in differentiation in some systems there has been no systematic study of PC expression in epidermis (2). We therefore investigated the expression and distribution of the members of the PC family in epidermis and examined the processing of several potential substrates.

In this study, 4 members of the PC family (furin, PACE4, PC5/6 and PC7/8) were detected in epidermis. Furin and PC7/8 were detected in distinct, although overlapping, patterns within the epidermis. In addition the active site of furin showed differential accessibility during differentiation. Inhibition of the PC family in culture inhibited the processing of Notch-1 and in vitro results characterized a furin/PACE4 processing site in the amino terminus of profilaggrin. The specific localization pattern, calcium dependence, and identification of possible substrates are consistent with a role for PCs in multiple events during epidermal differentiation.

\section{Methods}

\section{Keratinocyte culture}

Human foreskin keratinocytes were cultured using the methods described by Fleckman et al. (18), a modification of the Rheinwald-Green system. Cells were harvested for RNA or protein at either $\sim 60-70 \%$ of confluence, at $100 \%$ confluence or several days post-confluence.

\section{Detection of proprotein convertase expression in skin using degenerate primers}

The sequences of the known human PC members (furin, PACE4, PC1, PC2, PC5/6, PC7/8) were obtained from Genbank using the ENTREZ server. The sequences were multiply aligned using ClustalW $(19,20)$ and a highly conserved region of the catalytic domain was identified to which degenerate primers were designed. The sense primer $\left(5^{\prime}-\right.$ $\mathrm{CA}(\mathrm{C} / \mathrm{T}) \mathrm{GG}(\mathrm{C} / \mathrm{G}) \mathrm{AC}(\mathrm{A} / \mathrm{T} / \mathrm{G} / \mathrm{C})(\mathrm{A} / \mathrm{C}) \mathrm{G}(\mathrm{A} / \mathrm{T} / \mathrm{G} /$ C) TGTGC (A/T/G) GG-3') was based on the sequence $\mathrm{R}_{194} \mathrm{HGTRCAG}_{201}$ in furin which contains the active site arginine $\left(R_{194}\right)$. This sequence is $100 \%$ conserved across the known human members of the PC family and highly conserved in other species. The antisense primer $\left(5^{\prime}-\mathrm{GG}(\mathrm{T} / \mathrm{G})\right.$ CCC CAG CT (T/G) (C/G) (A/C) (A/G) CTG TA$3^{\prime}$ ) was based on the sequence $\mathrm{I}_{249} \mathrm{YSASWG}_{255}$ which is also highly conserved across the family, although in PC7/8 the alanine $\left(\mathrm{A}_{252}\right)$ is replaced by a cysteine. RT-PCR using these primers would amplify a 187 bp segment from each PC that could be distinguished on the basis of its nucleotide sequence.

Total RNA was isolated from 5-day post-confluent cultured human keratinocytes using the Trizol reagent (Life Technologies, Gaithersburg, MA, USA) and the manufacturer's protocol. After DNase I treatment the total RNA was reverse transcribed using the Superscript II RT system (Life Technologies) and the resulting cDNA was PCR amplified $\left(40\right.$ cycles at $95^{\circ} \mathrm{C}$ for $30 \mathrm{~s} ; 60^{\circ} \mathrm{C}$ for $1 \mathrm{~min} ; 72^{\circ} \mathrm{C}$ for $1 \mathrm{~min}$ ) using Taq plus Taq extender (Promega, Madison, WI, USA). The resulting $187 \mathrm{bp}$ product was gel purified and TA cloned into the pCR3.1 vector (Invitrogen, Carlsbad, CA, USA).

\section{Northern analysis}

Total RNA was isolated from human foreskin keratinocytes according to the method of Chomczynski \& Sacchi (21), fractionated on glyoxal gels, transferred to Genescreen Plus (Du Pont NEN, Boston, MA, USA) by capillary blotting according to manufacturer's recommendation, and hybridized with radiolabeled cDNA probes specific for the coding region of different members of the PC family $(22,23)$. The blots were subsequently hybridized with a GAPDH probe and the ratio between furin and GAPDH determined by Phosphorimager (Molecular Dynamics, Sunnydale, CA, USA). 


\section{PC expression and localization in epidermis}

\section{RT-PCR analysis of PC5/6 and PC7/8}

Oligoprimers specific for PC5/6 and PC7/8/LPC were made according to published sequences (Genbank acc\# NM-004716). For PC5/6 the sense primer used comprised nucleotides 962-982 (5'TCA GCA GCA CTG CAG AAA GC-3') and the antisense primer, nucleotides 1409-1429 (5'-TGG GGT CTT CAA CTC GGC TA-3'), giving a 447 bp product. For PC7/8 the sense primer 163-183 (5'-GCT GGT AGG CTG TTT CAC CG-3') and 667-647 (5'-GGA TTG CTG CCA GAG AAG TC-3') were used giving a 504 bp product. Reverse transcription was performed on the total RNA using the Superscript II kit (Life Technologies). PCR analysis was performed using 32 cycles of 94 ${ }^{\circ} \mathrm{C}, 1 \mathrm{~min} ; 50^{\circ} \mathrm{C}, 45 \mathrm{~s} ; 72^{\circ} \mathrm{C}, 1 \mathrm{~min}$ using Taq polymerase (Promega). PCR products were analyzed on a $1 \%$ agarose gel and their identity confirmed by sequence analysis.

\section{$P C$ protein expression in epidermis and keratinocytes}

Protein from foreskin epidermis and cultured human keratinocytes was extracted using a modified RIPA buffer (50mM HEPES, pH 7.5, $150 \mathrm{mM}$ $\mathrm{NaCl}, 50 \mathrm{mM} \mathrm{NaF}, 1 \% \mathrm{NP}-40,0.5 \%$ deoxycholate, $1 \mathrm{mM}$ sodium vanadate, $1 \mathrm{mM}$ nitrophenylphosphate, $5 \mathrm{mM}$ benzamidine, $2 \mathrm{mM}$ PMSF, $10 \mu \mathrm{g} / \mathrm{ml}$ aprotonin, $1 \times$ complete protease inhibitor (EDTA-free, Boehringer)) (24). The extracted proteins were separated on 7.5-12\% SDS-PAGE gels and blotted onto nitrocellulose for western immunoblot detection using specific antibodies and ECL. Furin was detected using a polyclonal antibody directed against the C-terminal tail (cfur) (a gift from Ruth Hogue Angeletti, New York University, USA) and monoclonal antibodies raised against catalytic domain (MON148) or cysteine-rich domain (MON152) epitopes (Alexis Biochemicals, San Diego, CA, USA). PC7/8 was detected using polyclonal antibodies directed against the catalytic and C-terminal domains (gift from Dr W. J. M. Van de Ven, Katholieke Universiteit Leuven, Belgium). PACE4 was detected using an antibody directed to the pro and catalytic domains (Alexis Biochemicals).

\section{Localization of furin in epidermis}

Immunofluorescence was performed on methanol fixed frozen or paraffin embedded methyl-Carnoy's fixed human epidermis. Carnoy's fixed tissues were first rehydrated and from then on all sections were treated the same. Antigen unmasking was performed on selected sections by incubation with
$0.1 \%$ trypsin for a variable length of time (usually $3 \mathrm{~min}$ ). After preblocking the sections with serum specific for the secondary antibodies used, the sections were incubated with the primary antibodies in TBS containing $1 \%$ BSA overnight at $4{ }^{\circ} \mathrm{C}$. The primary antibodies were detected with either FITC-conjugated secondary antibodies (Vector, Eugene, OR, USA) or biotin-conjugated secondary antibodies followed by streptavidin-conjugated Texas Red (Vector). DNA was stained with 4',6diamidino-2-phenylindole dihydrochloride (DAPI, $0.001 \%$ ). Sections were mounted in Prolong ${ }^{\circledR}$ mounting medium (Molecular Probes, Eugene, OR, USA). Images were obtained using a CCD camera attached to an epifluorescence Nikon SA microphot microscope.

\section{Inhibition of proprotein convertases in cultured keratinocytes}

Mouse epidermal keratinocytes were cultured to confluence as described previously (25). The PC inhibitor decanoyl-Arg-Val-Lys-Arg-chloromethylketone (Bachem, Torrence, CA, USA) (26) was added to the medium at feeding. Cells shed into the medium were collected by centrifugation and extracted first in RIPA buffer (24) and the pellet was subsequently re-extracted in $8.8 \mathrm{M}$ urea-Tris buffer (27). Equal amounts of protein were run on SDS-PAGE gels, blotted, probed with specific antibodies, including Notch-1 (a gift from L. Milner, Fred Hutchinson Cancer Research Center, Seattle, WA, USA) and visualized by ECL.

\section{In vitro cleavage of profilaggrin by furin and PACE4}

Preparation of furin and PACE4 enzymes. Transient transfectants of COS-1 or 293T cells with furin, mutant furin, and PACE4 expression constructs and with the control vector were performed as described elsewhere (22). Conditioned media from cells expressing soluble furin (furin), mutant soluble furin with the active site altered from serine to alanine (furin-SA), PACE4, and pMT3 (a control vector) $(22,23,28)$ were harvested and concentrated 10-fold using Centricon 10 (Amicon, Beverly, MA, USA) in 0.2 M sodium acetate $\mathrm{pH}$ 5.5. The concentrated media were stored in aliquots at $-80^{\circ} \mathrm{C}$ until use.

\section{Construction and expression of the profilaggrin substrate}

The cDNA expression construct used for these studies (referred to as pFLAG467proF) encodes the amino-terminal 467 amino acids of human 
profilaggrin, including the entire amino-terminal $\mathrm{A}+\mathrm{B}$ domains, plus the truncated filaggrin sequence. Preparation of the construct is described elsewhere (27). The construct encodes the 8 amino acid FLAG epitope at its amino-terminus. The profilaggrin cDNA construct was cloned into pcDNA3 (Invitrogen) for in vitro expression studies. COS-7 cells were transiently transfected using LipofectAMINE (Life Technologies) with $5.5 \mu \mathrm{g}$ of the profilaggrin cDNA constructs. Cells were usually harvested $48 \mathrm{~h}$ after transfection and lysates prepared in $0.25 \mathrm{M}$ Tris/ $\mathrm{HCl}, \mathrm{pH} 7.8$, using three freeze/thaw cycles in dry ice-ethanol and 37 ${ }^{\circ} \mathrm{C}$ water baths. Solubilized cell extracts were clarified by centrifugation at 12,000 r.p.m. for $10 \mathrm{~min}$ and stored frozen in aliquots until use.

\section{Site-directed in vitro mutagenesis}

Site directed mutagenesis on double-stranded pFLAG467proF DNA was performed using the method based on the QuikChange mutagenesis kit (Stratagene, LaJolla, CA, USA) to render a nonconservative mutation, either at $\mathrm{P} 1, \mathrm{P} 2$, or $\mathrm{P} 4$ of the cleavage site, from Arg/Lys to Ala. The three mutagenic oligonucleotide primers (Life Technologies) were as follows ( $5^{\prime}$ to $3^{\prime}$ ) (mutated bases are underlined, and the amino acid changes produced at the cleavage site are shown in brackets):

P1/Ala,

$$
\begin{aligned}
& \text { GGACAAGAAAGCGTGCGGGATCCA } \\
& \text { GAGTTAGC (RKRA); } \\
& \text { P2/Ala, } \\
& \text { GGACAAGAAAGGCTAGGGGATCCA } \\
& \text { GAGTTAGC (RKAR); } \\
& \text { P4/Ala, } \\
& \text { GGAGTCCAGGA } \\
& \text { CAGCAAAGCGTAGGGG (AKRR). }
\end{aligned}
$$$$
\text { P4/Ala, }
$$

The altered PC cleavage sites were confirmed by sequencing the mutated constructs.

\section{Proprotein convertase cleavage assay and immunoblotting procedure}

COS-7 cell extracts containing the substrate were incubated with conditioned or mock medium (enzyme or control prep) at $37^{\circ} \mathrm{C}$ for $16 \mathrm{~h}$ in a final volume of $50 \mu \mathrm{l}$ of $0.1 \mathrm{M}$ HEPES buffer ( $\mathrm{pH} 7.0$ ), $1 \mathrm{mM} \beta$-mercaptoethanol, and $5 \mathrm{mM} \mathrm{CaCl}_{2}$. The reaction was terminated by the addition of SDSPAGE sample buffer and boiled before loading onto a $12 \%$ or $12-15 \%$ gradient SDS-polyacrylamide gel. The gel was electroblotted to nitrocellulose and the amino-terminal domain of profilaggrin identified using the polyclonal anti-B1-peptide antibody described previously (specific for the B domain) (27).
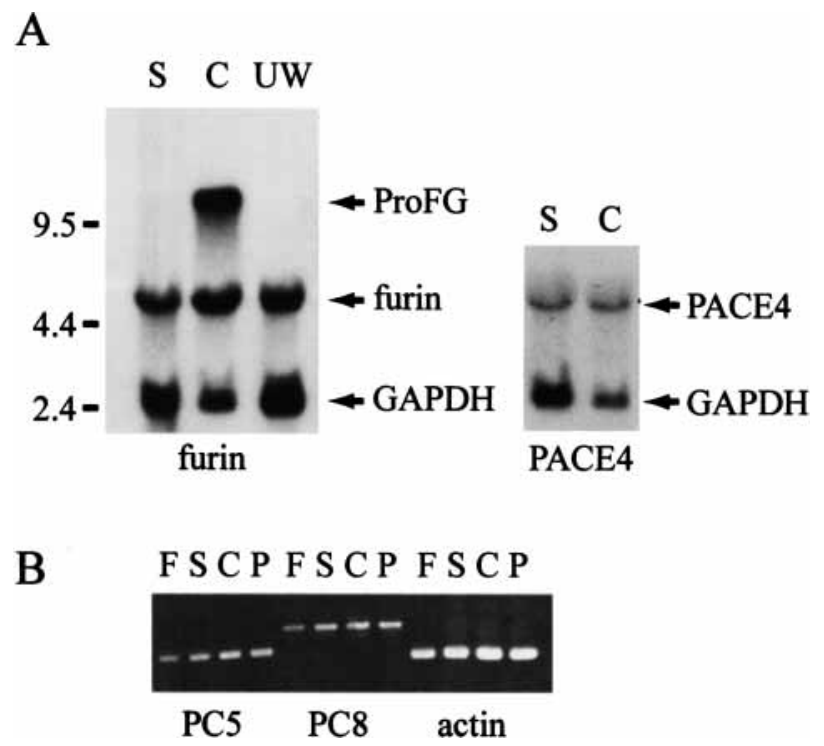

Figure 1. Expression of PC family members in cultured human keratinocytes and epidermis. (A) Northern analysis of total RNA from subconfluent (S) and confluent (C) cultured human keratinocytes. Medullo blastoma cell line (UW) was used as a positive control for furin/PACE expression. RNA was fractionated on a $1 \%$ glyoxal gel, blotted, and hybridized with DNA probes specific for furin/PACE, PACE4, and profilaggrin. The blots were subsequently hybridized with glyceraldehyde-3-phosphate dehydrogenase (GAPDH) to normalize the amount of RNA. Molecular weight markers are designated on the left. Both subconfluent and confluent (profilaggrin expressing) keratinocytes expressed a $5 \mathrm{~kb}$ furin message and a similar sized PACE4 transcript. The furin message was increased 3-fold in confluent vs subconfluent cells. (B) RT-PCR analysis of total RNA from foreskin epidermis (F) and from subconfluent (S), confluent (C), and postconfluent (P) keratinocytes cultures. Primers specific for PC5/6, PC8, and actin were used. Both cells and tissue had detectable levels of PC5/6 and PC7/8.

\section{Results}

Members of the proprotein convertase family are expressed in human keratinocytes

To determine if human epidermal keratinocytes express members of the PC family we analyzed mRNA expression using degenerate primers directed towards the active site of the PC family. Cloning and sequencing of multiple products resulted in identification of furin, PACE4, PC5/6 and $\mathrm{PC} 7 / 8$. In order to confirm this expression pattern we used Northern blot and RT-PCR analysis of epidermal and cultured human keratinocyte RNA. Northern analysis of total RNA from human keratinocytes at subconfluence and confluence using cDNA probes specific to human furin/PACE and PACE4 shows hybridization signals with the molecular size of approximately $5 \mathrm{~kb}$ (Fig. 1A); similar to the size of these mRNAs in other cells and tissues (12). Quantitative Phosphorimager analysis of the level of furin/PACE expression relative to 
GAPDH indicates that the level increased approximately 3-fold upon confluence. GAPDH expression levels are unchanged in pre vs postconfluent cells (29). Similar results were obtained with PACE4 expression (Fig. 1A). As a control for changes in keratinocyte gene expression with confluence, the level of human profilaggrin mRNA was evaluated. Profilaggrin expression increased dramatically in the confluent cultures (Fig. 1A), consistent with previous results (29). In addition, mRNAs for PC5/6 and PC7/8/LPC were detected by RT-PCR amplification using specific primers (Fig. 1B) both in cultured keratinocytes and foreskin epidermis; the identity of these products was confirmed by sequencing. Probes corresponding to PC1/PC3, PC2, and PC4 were tested by Northern blotting but did not hybridize to keratinocyte RNA under the conditions used, nor were they detected in RT-PCR experiments (data not shown), in agreement with previous evidence suggesting that these PC family members are specific to other cell types or tissues (12). Thus, we have evidence for expression of four members of the PC family in epidermal keratinocytes.

\section{Proprotein convertase protein expression in epidermis and keratinocytes}

Antibodies to the catalytic domain and cytoplasmic domains of both furin and PC7/8 were used to further examine expression and localization of these proteins in epidermis (Figs 2, 3 \& 4).

The $97 \mathrm{kDa}$ mature furin was detected in foreskin epidermal extracts and in subconfluent and postconfluent keratinocytes (Fig. 2A). The $97 \mathrm{kDa}$ protein was recognized by antibodies to the C-terminal cytoplasmic domain (c-fur), catalytic domain (MON148) and the cys-rich domain (MON152) indicating that it corresponds to the mature enzyme after proteolytic removal of the prepro domain. This band corresponds to the size of a furin expressed in COS cells via a cDNA con-

A
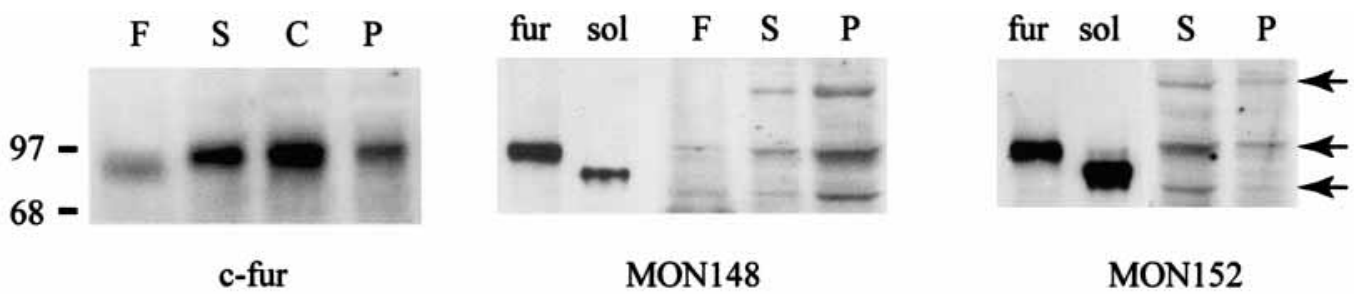

MON148

MON152

B

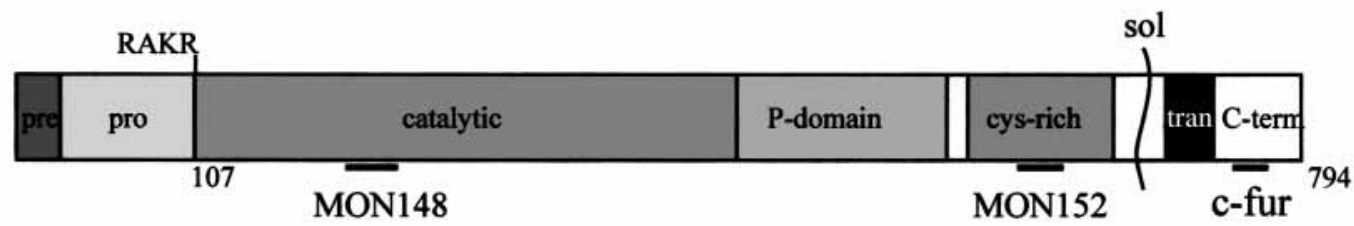

C
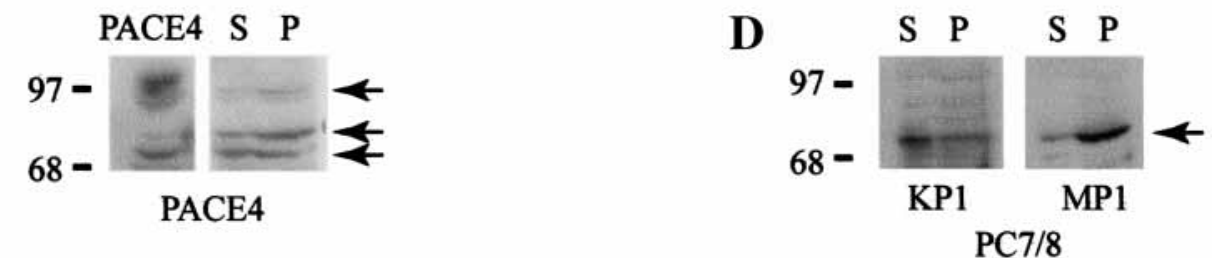

Figure 2. Western blots of PC expression in extracts of foreskin epidermis and cultured keratinocytes. Foreskin (F) and subconfluent (S), confluent (C) and postconfluent (P) keratinocyte extracts were separated on 7.5-12\% SDS-PAGE gels, transferred to nitrocellulose and probed with specific antibodies using the ECL system. The positions of molecular weight standards are indicated on the left. (A) Expression of furin. Antibody c-fur, specific for the C-terminal cytoplasmic domain, recognized a $97 \mathrm{kDa}$ band in epidermal and keratinocyte extracts. Antibodies directed against the active site (MON148) and cysteine-rich domain (MON152) recognized 97,75 , and $120 \mathrm{kDa}$ bands (arrows), although the $120 \mathrm{kDa}$ band was not detected in epidermal extracts. The $97 \mathrm{kDa}$ band is equivalent in size to the expressed furin cDNA construct from COS cells (lane fur in MON 148 and MON 152 blots), while the 75 $\mathrm{kDa}$ band is slightly smaller in size than the soluble furin construct lacking the transmembrane and C-terminal domain (lane sol in MON 148 and MON 152 blots). The $120 \mathrm{kDa}$ band detected in keratinocytes may correspond to the proenzyme. (B) Diagram of furin indicating epitopes for the antibodies used in A. The transmembrane domain is shown in black and the position of the protein truncation to produce the soluble form (sol) is shown by the waved line. (C) Expression of PACE4. Bands of $95 \mathrm{kDa}, 75 \mathrm{kDa}$ and $70 \mathrm{kDa}$ (arrows) were recognized by the PACE4 polyclonal antibody in control PACE4 expressed in 293T cells (PACE4) and subconfluent (S) and postconfluent (P) keratinocytes. (D) Expression of PC7/8. The active $75 \mathrm{kDa}$ protein (arrow) is detected in both subconfluent and postconfluent cells using antibodies to both the C-terminus (KP1) and the N-terminus (MP1). 


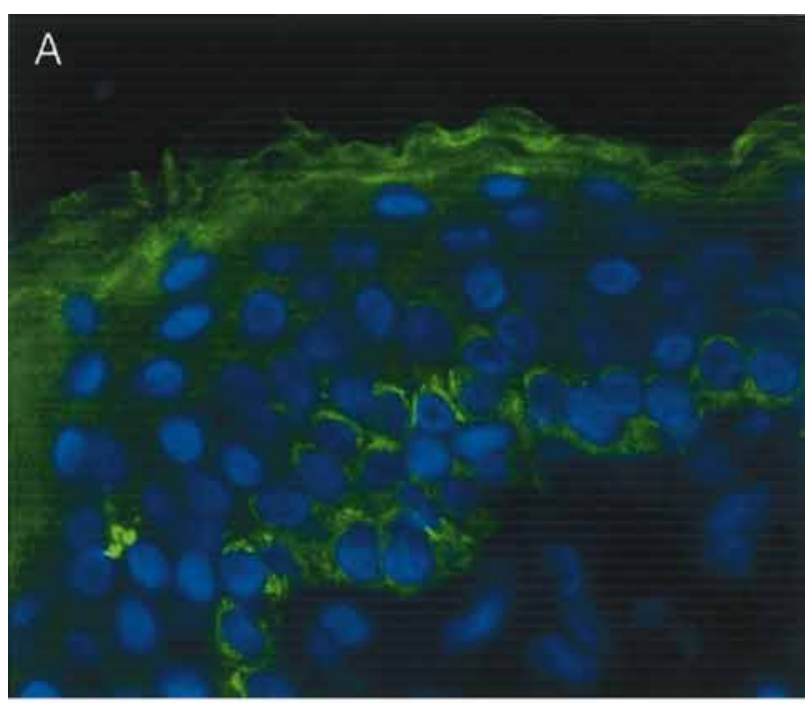

fur

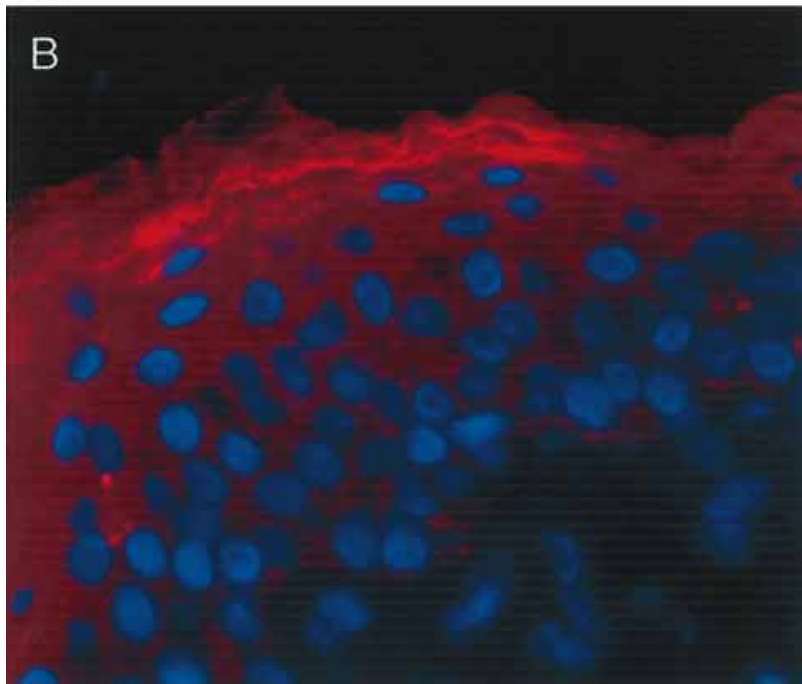

Mon148

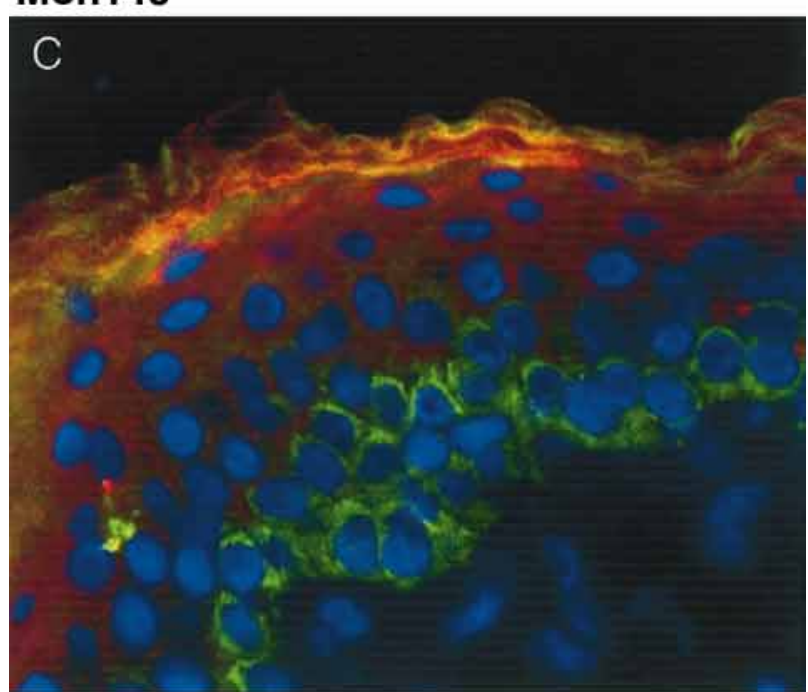

Merged
Figure 3. Double immunofluorescence of epidermis using the cfur and MON148 antibodies. (A) c-fur reaction. (B) MON148 reaction. (C) Merged c-fur and MON148 reactions; the yellow color indicates where the green and red signals are superimposed to give yellow fluorescence. Tissue sections were trypsin treated $(0.01 \%, 1 \mathrm{~min})$ and incubated overnight $\left(4^{\circ} \mathrm{C}\right)$ with the rabbit polyclonal c-fur antibody and the mouse monoclonal MON148 antibody. The c-fur antibody was detected with a FITC-labeled anti-rabbit secondary antibody (green); the MON148 was detected using a biotinylated anti-mouse secondary/Texas Red streptavidin ternary complex (red); DNA in nuclei was stained with DAPI (blue). The C-terminal antibody (c-fur, A \& C) stains both the basal and granular layer with significantly reduced staining in the spinous layer. The catalytic domain antibody (MON148, B \& C) stains mainly the granular layer with lighter spinous and very little basal staining. In the granular layer the merged staining $(\mathrm{C})$ shows both co-localization (yellow) as well as patches of differential localization of the C-terminus (green) and catalytic (red) domains.

struct. An additional $70-75 \mathrm{kDa}$ band was observed in epidermis and keratinocytes that reacted with antibodies to the catalytic domain (MON148) and cys-rich domain (MON152), but not the Cterminal cytoplasmic domain (c-fur). This band is slightly smaller than soluble furin produced from a construct lacking the transmembrane and C-terminal domains, indicating cleavage and removal of the transmembrane domain in the tissue. A larger band $(\sim 120 \mathrm{kDa})$, possibly corresponding to the proenzyme, was detected in keratinocytes but not in foreskin epidermal extracts. The specificity and identity of the bands recognized by the antibodies was confirmed by stripping representative blots and reprobing with an alternate antibody (data not shown).

Three bands (96 kDa, $75 \mathrm{kDa}$ and $70 \mathrm{kDa}$ ) positive for PACE4 were detected on western blots in sub- and post-confluent keratinocyte extracts (Fig. 2C). PC7/8 protein was also detected in pre- and post-confluent keratinocytes (Fig. 2D). The active form at $\sim 75 \mathrm{kDa}$ was seen in both pre- and postconfluent cell extracts with both antibodies.

\section{Localization of proprotein convertases in epidermis}

Furin staining was detected by immunofluorescent localization within the epidermis. The antibody to the cytoplasmic domain (c-fur) showed strong staining in the basal and granular layers with less staining in the spinous layers (Fig. 3A). In contrast the antibody against the active site (MON148) showed the greatest concentration of staining in the granular layer (MON148; Fig. 3B), with less staining in the lower layers of the epidermis. The staining was strongly cytoplasmic. In the granular layer the staining pattern for the cytoplasmic domain (c-fur) and catalytic domain (MON148) were not completely correlated (Fig. 3C). Areas of yel- 


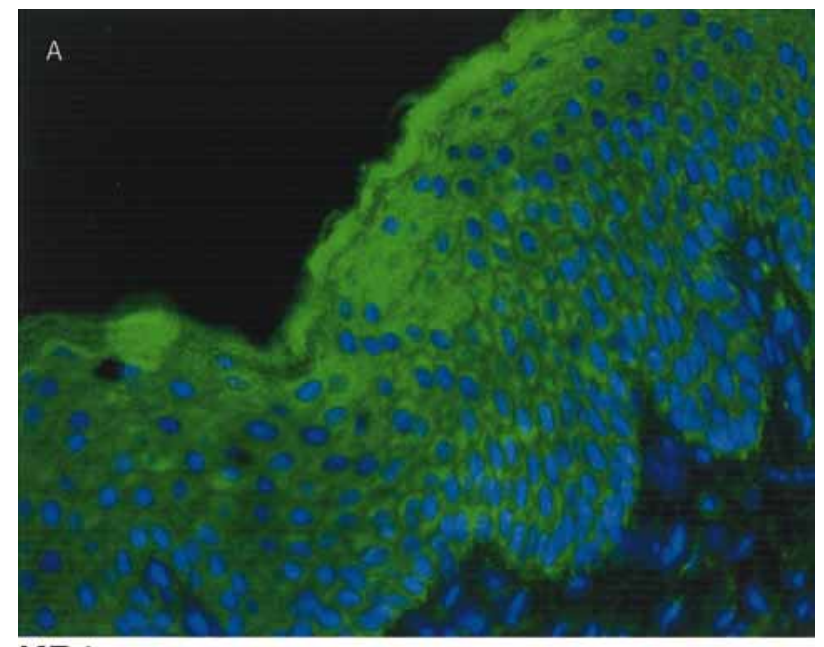

\section{KP1}

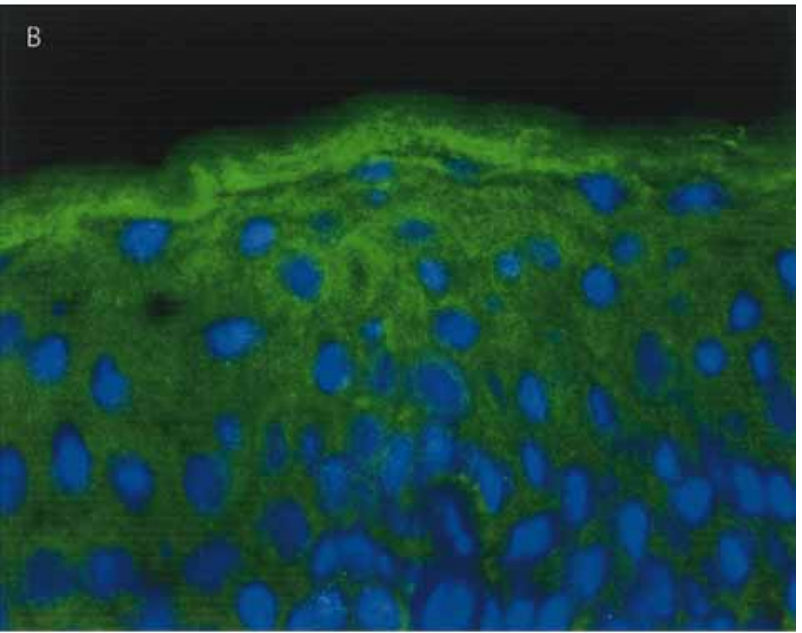

\section{MP1}

Figure 4. Immunofluorescence of epidermis using antibodies specific for PC7/8. (A) Staining with the antibody KP1 which is directed at an epitope in the C-terminal cytoplasmic domain. (B) Staining with antibody MP1 which recognizes the N-terminus of the catalytic domain. Tissue sections were incubated with the polyclonal KP1 or MP1 (1 h, RT) and detected using a FITC-labeled anti-rabbit secondary (green). Nuclei were stained with DAPI (blue). Both antibodies stained all layers of the epidermis and were confined to the cytoplasm of the cells. Note the slight increase of staining in the granular layer.

low in the granular layer indicate where the red MON148 staining and green c-fur staining are colocalized (merged). There are, however, significant areas where the two antibodies are not superimposed (red and green staining in the granular layer). Strong furin staining was also seen in the glandular structures of the dermis (not shown).

Antibodies specific for PC7/8 showed patchy staining throughout the epidermis (Fig. 4). This distribution was similar with both the antibody directed against the $\mathrm{C}$-terminal cytoplasmic domain (KP1) (Fig. 4A) and that directed against the catalytic domain (MP1) (Fig. 4B).

\section{Day \\ [RVKR-CMK] $0 \longdiv { 0 1 0 0 2 5 0 }$}

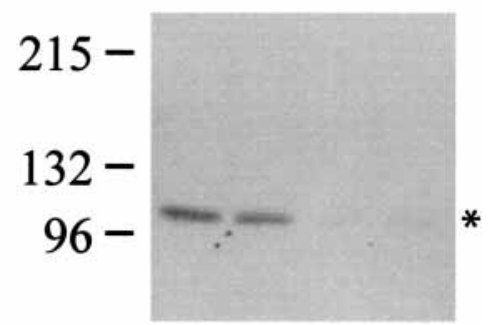

Figure 5. Inhibition of Notch-1 processing in mouse keratinocytes by decanocyl-RVKR-CMK. Mouse keratinocytes were treated with the inhibitor or vehicle for 4 days. Cell extracts were separated on a 7.5-15\% SDS-PAGE gel, blotted onto nitrocellulose and probed with a monoclonal anti-Notch-1 antibody directed to an epitope in the intracellular C-terminal domain using the ECL system. The $120 \mathrm{kDa}$ active Notch-1 amino-terminal domain $\left({ }^{*}\right)$ is detected in the pretreated cultures (day 2) as well as in the day 4 cultures without the PC inhibitor but is significantly reduced in cultures treated with $100 \mu \mathrm{M}$ and $250 \mu \mathrm{M}$ of the inhibitor. The position of molecular weight standards is shown on the left. The Notch-1 pro-form is not detected due to its poor reactivity with the antibody (Milner, personal communication).

\section{Identification of potential PC substrates in epidermis}

Two potential substrates, Notch-1 and profilaggrin, relevant in epidermal differentiation, were investigated using either in vivo or in vitro approaches.

\section{Inhibition of Notch-1 processing by inhibition of proprotein convertase processing in culture}

Notch-1 is a cell-surface receptor involved in cell fate determination and patterning. Processing of Notch-1 by furin at an RXRR site in the carboxy terminus during transport to the plasma membrane is required to produce the functional receptor $(14,30)$. A cell permeable PC inhibitor (decanoyl-RVKR-CMK) (26) was added to mouse keratinocyte cultures to test the role of furin on Notch-1 processing in these cells. Inhibition of the processing of Notch-1 from the precursor form $(220 \mathrm{kDa})$ to the functional $120 \mathrm{kDa}$ form (Fig. 5) was seen with dramatic reduction of the $120 \mathrm{kDa}$ form using both 100 and $250 \mu \mathrm{M}$ of the inhibitor. The inhibitor did not exhibit toxic effects on the cells at these concentrations.

\section{Furin and PACE4 specifically cleave the amino terminus of profilaggrin}

Profilaggrin is a large $(>400 \mathrm{kDa})$ protein, comprised of multiple filaggrin domains flanked by 
unique $\mathrm{N}$ and $\mathrm{C}$-termini, that is expressed in the granular layer of the epidermis (reviewed in 31). Normal keratinocyte culture systems can be induced to produce profilaggrin $(18,29,32)$ but do not fully process it into filaggrin and the aminoterminal fragment. Therefore, to study potential PC processing of profilaggrin at the predicted sequence $\left(R_{288} T_{R K R} R_{293}\right)$ at the junction of the amino terminus and the filaggrin domains, an in vitro system was used. Extracts obtained from COS-7 cells transfected with the N-terminal profilaggrin expression construct, pFLAG467proF, were used to mimic profilaggrin; profilaggrin itself is insoluble in aqueous buffers suitable for enzyme assays and therefore could not be used in these assays. Media conditioned by COS- 1 or $293 \mathrm{~T}$ cells transfected with expression constructs encoding either a soluble form of furin, its inactive mutant derivative (furin-SA), or PACE4 were sources of the enzymes. Cleavage of the substrate was de-

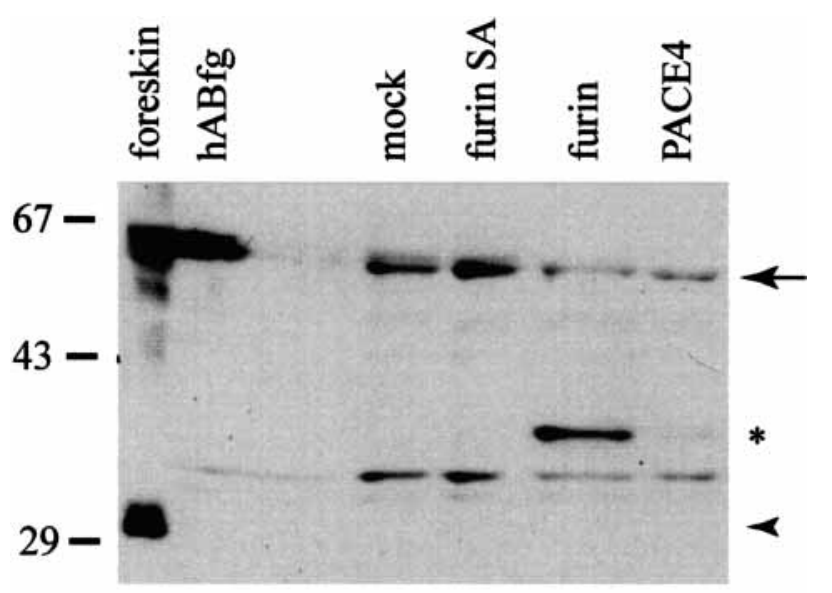

\section{FLAG-hABfg}

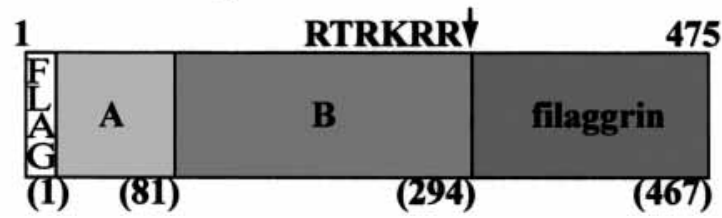

Figure 6. Cleavage of profilaggrin (pFLAG467proF) by furin and PACE4. Extracts of COS-7 cells transfected with the pFLAG467proF were incubated with conditioned media containing expressed enzyme. The products were analyzed using antibody specific for the B domain of the profilaggrin aminoterminus. The position of uncleaved product is shown by the arrow. Note that a cleaved product (approx. $37 \mathrm{kDa}^{*}$ ) is detected in the samples containing furin (strong) and PACE4 (weak), but not those containing an inactive mutant (furin-SA) or mock transfected media or the unreacted substrate (hABfg). The endogenous profilaggrin $\mathrm{N}$-terminus $(32 \mathrm{kDa})$ is visible in the foreskin extract (arrowhead). Molecular weight markers are indicated on the left. A diagram of the FLAG467proF construct is shown to illustrate the A and B domains and the position of the predicted cleavage site. tected by the appearance of the processed fragment on western blots using antibodies specific for the $\mathrm{B}$ domain of the profilaggrin $\mathrm{N}$-terminus. The antibody specific for the amino-terminus reacts with both the amino-terminal-profilaggrin substrate and the expected cleavage product (Fig. 6), whereas the antibody specific for filaggrin reacts only with the amino-terminal-profilaggrin substrate and not with the cleaved product (data not shown). As shown in Fig. 6, only treatment of the profilaggrin substrate with the soluble form of furin or with PACE4 but not with the mutant furin (furin-SA) resulted in the appearance of a new band representing the proteolytic cleavage product with a size of approximately $37 \mathrm{kDa}$. This cleavage is $\mathrm{Ca}^{2+}$-dependent and can be inhibited with EDTA (data not shown). Control substrate (von Willebrand factor or CPA-PACE) was used to verify that the enzyme preparations functioned as expected (data not shown).

In order to confirm the specific site of PC cleavage, the proposed cleavage site $\left(\mathrm{R}_{288} \mathrm{TRKRR} \mathrm{R}_{293}\right)$ was mutated to see if specific processing could be affected. Fig. 7 shows that substitution at either the P1 position (to RKRA) or the P4 position (to AKRR) virtually abolished the cleavage which produces the $37 \mathrm{kDa}$ product in the normal construct, whereas the alteration of the $\mathrm{P} 2$ position (to RKAR) had little effect on the cleavage. The results demonstrated that the predicted paired amino acid sequence is required as the primary target site

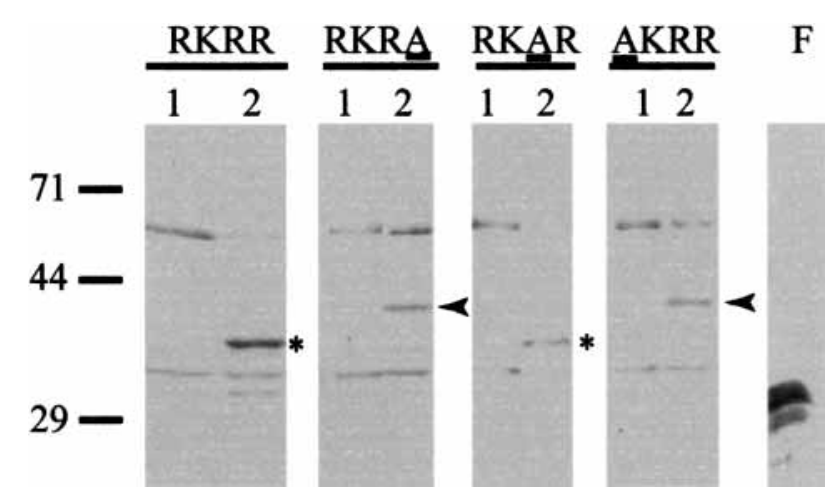

Figure 7. Effect of mutation at residues $\mathrm{P} 1, \mathrm{P} 2$, and $\mathrm{P} 4$ of the putative profilaggrin cleavage site. COS-7 cells were transfected with the profilaggrin expression construct carrying either the parental (RKRR) or one of the mutant cleavage sites (RKRA, RKAR, AKRR) and the extracts were treated with conditioned media containing mock media (lane 1) or soluble furin (lane 2). The $37 \mathrm{kDa}$ cleaved product is visible in the furin cleaved-parental construct and the RKAR mutant and was identified using the antibody specific for the profilaggrin B-domain $(*)$. The normal $37 \mathrm{kDa}$ cleavage product is absent from the RKRA and AKRR mutants although a secondary cleavage product at 40 $\mathrm{kDa}$ is seen (arrowhead). Molecular weights are indicated on the left. Lane $\mathrm{F}$ is human foreskin extract run as a control on the same gel showing the $32 \mathrm{kDa}$ endogenous cleavage product. 
for furin/PACE cleavage in vitro. Interestingly, mutagenesis of each of the critical residues (P1, P4) yielded another cleaved product migrating at about $40 \mathrm{kDa}$. This product could be either an intermediate of a sequential cleavage or a product resulting from an alternative secondary site, possibly the sequence GSRHPR present at residues 331-336.

\section{Discussion}

The dramatic remodeling of cytoplasm and cell membrane that occurs at the transition of the granular and cornified layers to form the protective skin surface requires multiple proteolytic events, breakdown of nucleic acids and membranebounded cellular organelles, and restructuring of the keratin cytoskeleton and plasma membrane. The changes in keratinocytes during the basal to spinous layer transition are not as dramatic morphologically yet regulate the balance between proliferation and withdrawal from the cell cycle and differentiation. The results presented here show that members of the PC family are likely to play a role in these events, consistent with their involvement in differentiation of gut epithelia and other tissues (33).

We have shown the expression in epidermis of four PC family members; furin, PACE4, PC5/6 and $\mathrm{PC} 7 / 8$ at the mRNA level and detected protein for three of these proteases. Furin, in particular, is detected in two forms - the mature $97 \mathrm{kDa}$ form and a smaller $75 \mathrm{kDa}$ form that lacks reactivity with the c-fur antibody specific for the $\mathrm{C}$-terminus. Based on size and antibody reactivity, the $75 \mathrm{kDa}$ form is expected to lack the transmembrane domain. This size difference may be associated with the change in the accessibility of furin in the different layers of the skin shown by immunocytochemistry using antibodies directed to specific epitopes. The cytoplasmic C-terminal domain is detected in both the basal (less differentiated) layer and the granular layer whereas the catalytic domain is most readily detected in the granular layer where cells are preparing to undergo terminal differentiation. This implies that the catalytic domain is more accessible to the antibody in the granular cells. As the cells of the granular layer are undergoing significant remodeling, including the dissolution of membranous organelles and the remodeling of the golgi/transgolgi apparatus into lamellar granules (34-36), it is likely that there is some rearrangement of the normal contents of these organelles, including the normally trans-golgi resident furin, which would allow it access to a wider range of substrates in these cells.

These results suggest that furin might play multiple roles in epidermal differentiation depending on the cell layer. Thus in the basal layer it would be expected to be involved in processing of secreted substrates including collagen XVII, integrins $\alpha 3$ and $\alpha 6$, and Notch, which mature via the golgi-transgolgi route. Our studies clearly show that inhibition of furin (and other PCs) in keratinocytes blocked the processing of Notch-1, a cell surface receptor that has been shown to be involved in cell fate determination and patterning (37-41) and integrin signaling (17). Notch is expressed in all layers of the epidermis and has been suggested to have a role in stem cell fate and maintenance in epidermis (15) and hair follicles $(42,43)$. The potential importance of the Notch pathway is emphasized by the expression of the fringe genes, modulators of Notch signaling, in specific patterns in epidermis (44). Furin over-expression has been shown to inhibit differentiation in $\alpha$-cells of pancreatic islets (45) and gastric mucosal cells (33). In contrast inhibition of furin in epidermal organotypic cultures leads to premature differentiation of keratinocytes, possibly via its effects on the Notch pathway (Pearton et al., in preparation).

Furin is not strongly expressed in the spinous layers, but is detectable in the granular layer with altered epitope accessibility correlated with detection of the $75 \mathrm{kDa}$ form of the enzyme. In this layer furin, and other PCs, would have access to a different range of substrates including cytoplasmic proteins such as profilaggrin. Both furin and PACE4 are able to cleave profilaggrin in vitro to release a fragment of the size predicted by cleavage at the junction with the filaggrin domains (27). PC5/6 and PC7/8 are also expressed in the epidermis but it is not known whether they have similar cleavage specificity towards the profilaggrin substrate.

Profilaggrin is a highly phosphorylated insoluble protein of keratohyalin. During terminal differentiation it is processed by phosphatases and proteases to release the amino-terminus and individual filaggrin domains which bind to and aggregate keratin filaments (46). Although several enzymes involved in profilaggrin processing are known, the early events are not understood (reviewed in 31). The amino-terminus containing the $\mathrm{S}-100 \mathrm{Ca}^{2+}$-binding domain (A domain) and a cationic $\mathrm{B}$ domain moves to the nucleus in transition cells while the filaggrin domains remain in the cytoplasm (47) $(27,48)$. A canonical PC cleavage motif $\left(R_{288} T_{R K R R_{293}}\right)$ lies at the junction between the $\mathrm{B}$ domain and the first filaggrin repeat. This site is very different from the hydrophobic cleavage motif (FLY in human) located between the filaggrin repeats $(5,49)$ which is the target of specific proteases such as PEP1 (5). The domain- 
specific cleavage of profilaggrin in vitro $(37 \mathrm{kDa}$ product) is similar but not identical to that detected in vivo (approx. $32 \mathrm{kDa}$ ) (Figs 6, 7). This difference could be due in part to the presence of the FLAG epitope which was required to enhance solubility of this test substrate. However, additional cleavages may also occur in vivo, and further study will be required to determine the involvement of $\mathrm{PC}$ in this processing pathway in vivo.

Nevertheless, cleavage of the profilaggrin amino-terminus is a critical event for altering the solubility of the protein for subsequent PEP1 cleavage of the filaggrin units (Resing et al., submitted), as well as for generating the S-100-like domain for nuclear translocation where it may have distinct functions in calcium-regulated events during terminal differentiation $(47,48,50,51)$. The profilaggrin domain-specific processing, the furin localization and its change in accessibility during differentiation, and the calcium-dependent processing, all suggest a functional role for PCs in terminal differentiation in vivo. Our findings emphasize the pivotal role of proteases in processing profilaggrin and adds furin/PACE to the list of proteases (i.e. profilaggrin endoproteinase 1 (5), calpain (52), plasmin and chymotrypsin-like proteases (53)) that are likely to have significant roles in terminal differentiation of epidermal cells.

In summary we present the first systematic analysis into the expression and localization of the PCs in epidermis. We have shown the localization of furin and PC7/8 using immunofluorescence and present evidence that furin undergoes a change of accessibility between the basal and granular layers that is related to cleavage to a soluble form that would have access to different types of substrates in these two distinct sites. We show that inhibition of PCs in keratinocytes inhibits processing of Notch-1, a known substrate with implications for cell fate determination and patterning. We also demonstrate that PCs are able to specifically cleave the amino-terminus of profilaggrin, a novel substrate found in the granular layer of the epidermis. This work indicates that the PCs may play multiple roles during the differentiation of cells within the epidermis.

\section{Acknowledgements}

We thank Dr Michael Bobola for the UW 228-1 cell line, Janet Kimball for her assistance with immunoblotting, Barbara Hager for her expertise in tissue culture, Dr Ruth Houge Angeletti (Albert Einstein College of Medicine, NY) for the gift of the anti-furin antibody, Dr W.J.M. Van de Ven (Katholieke Universiteit Leuven, Belgium) for the gift of the anti-PC7/8 antibody and Dr Lauri Milner (Fred Hutchinson Cancer Research Center, Seattle, WA, USA) for the gift of the Notch-1 antibody. Tissue culture procedures were performed in the Keratinocyte
Culture Core Laboratory, Dr Philip Fleckman, Director, and immunostaining was photographed in the Morphology Core Laboratory, Dr Micheal Piepkorn, Director, Division of Dermatology with assistance from Mr Robert Underwood, part of the Dermatology Program Project, P01 AR21557. Autoradiographic analysis was carried out by the PhosphorImager facility of the Markey Molecular Medicine Center at the University of Washington. This work was supported by NIH grants R39 DE04660 and P01 AR21557 to BAD. R.B. Presland was the recipient of a Career Development Award from the Dermatology Foundation.

\section{References}

1. Kam E, Resing K A, Lim S K, Dale B A. Identification of rat epidermal profilaggrin phosphatase as a member of the protein phosphatase 2A family. J Cell Sci 1993: 106: 219.

2. Hart T C, Hart P S, Bowden D W et al. Mutations of the cathepsin $\mathrm{C}$ gene are responsible for Papillon-Lefevre syndrome. J Med Genet 1999: 36: 881.

3. Kim S Y, Bae C D. Calpain inhibitors reduce the cornified cell envelope formation by inhibiting proteolytic processing of transglutaminase 1. Exp Mol Med 1998: 30: 257.

4. Tanabe H, Kumagai N, Tsukahara T et al. Changes of lysosomal proteinase activities and their expression in rat cultured keratinocytes during differentiation. Biochim Biophys Acta 1991: 1094: 281 .

5. Resing K A, Johnson R S, Walsh K A. Characterization of protease processing sites during conversion of rat profilaggrin to filaggrin. Biochemistry 1993: 32: 10036.

6. Watkinson A. Stratum corneum thiol protease (SCTP) a novel cysteine protease of late epidermal differentiation. Arch Dermatol Res 1999: 291: 260.

7. Hart T C, Hart P S, Michalec M D et al. Haim-Munk syndrome and Papillon-Lefevre syndrome are allelic mutations in cathepsin C. J Med Genet 2000: 37: 88.

8. Toomes C, James J, Wood A J et al. Loss-of-function mutations in the cathepsin $\mathrm{C}$ gene result in periodontal disease and palmoplantar keratosis. Nat Genet 1999: 23: 421.

9. Creemers J W, Jackson R S, Hutton J C. Molecular and cellular regulation of prohormone processing. Semin Cell Dev Biol 1998: 9: 3.

10. Gensberg K, Jan S, Matthews G M. Subtilisin-related serine proteases in the mammalian constitutive secretory pathway. Semin Cell Dev Biol 1998: 9: 11.

11. Nakayama K. Furin: a mammalian subtilisin/Kex2p-like endoprotease involved in processing of a wide variety of precursor proteins. Biochem J 1997: 327 (Pt 3): 625.

12. Seidah N G, Chretien M. Pro-protein convertases of subtilisin/kexin family. Methods Enzymol 1994: 244: 175.

13. Krysan D J, Rockwell N C, Fuller R S. Quantitative characterization of furin specificity: Energetics of substrate discrimination using an internally consistent set of hexapeptidyl methylcoumarinamides. J Biol Chem 1999: 274: 23229.

14. Logeat F, Bessia C, Brou C et al. The Notch1 receptor is cleaved constitutively by a furin-like convertase. Proc Natl Acad Sci USA 1998: 95: 8108.

15. Lowell S, Jones P, Le Roux I, Dunne J, Watt F M. Stimulation of human epidermal differentiation by delta-notch signalling at the boundaries of stem-cell clusters. Curr Biol 2000: 10: 491.

16. Schacke H, Schumann H, Hammami-Hauasli N, Raghunath M, Bruckner-Tuderman L. Two forms of collagen XVII in keratinocytes. A full-length transmembrane protein and a soluble ectodomain. J Biol Chem 1998: 273: 25937.

17. Berthet V, Rigot V, Champion S et al. Role of endoproteolytic processing in the adhesive and signalling functions of alphavbeta5 integrin. J Biol Chem 2000: 275: 33308.

18. Fleckman P, Holbrook K A, Dale B A, Sybert V P. Kera- 


\section{PC expression and localization in epidermis}

tinocytes cultured from subjects with ichthyosis vulgaris are phenotypically abnormal. J Invest Dermatol 1987: 88: 640.

19. Thompson J D, Higgins D G, Gibson T J. CLUSTAL W: improving the sensitivity of progressive multiple sequence alignment through sequence weighting, position-specific gap penalties and weight matrix choice. Nucleic Acids Res 1994: 22: 4673 .

20. Higgins D G, Thompson J D, Gibson T J. Using CLUSTAL for multiple sequence alignments. Methods Enzymol 1996: 266: 383.

21. Chomczynski P, Sacchi N. Single-step method of RNA isolation by acid guanidinium thiocyanate- phenol-chloroform extraction. Anal Biochem 1987: 162: 156

22. Rehemtulla A, Dorner A J, Kaufman R J. Regulation of PACE propeptide-processing activity: requirement for a post-endoplasmic reticulum compartment and autoproteolytic activation. Proc Natl Acad Sci U S A 1992: 89: 8235.

23. Rehemtulla A, Barr P J, Rhodes C J, Kaufman R J. PACE4 is a member of the mammalian propeptidase family that has overlapping but not identical substrate specificity to PACE. Biochemistry 1993: 32: 11586.

24. Aplin A E, Juliano R L. Integrin and cytoskeletal regulation of growth factor signaling to the MAP kinase pathway. J Cell Sci 1999: 112: 695.

25. Hager B, Bickenbach J R, Fleckman P. Long-term culture of murine epidermal keratinocytes. J Invest Dermatol 1999: 112: 971

26. Garten W, Hallenberger S, Ortmann D et al. Processing of viral glycoproteins by the subtilisin-like endoprotease furin and its inhibition by specific peptidylchloroalkylketones. Biochimie 1994: 76: 217.

27. Presland R B, Kimball J R, Kautsky M B, Lewis S P, Lo C Y, Dale B A. Evidence for specific proteolytic cleavage of the N-terminal domain of human profilaggrin during epidermal differentiation. J Invest Dermatol 1997: 108: 170.

28. Rehemtulla A, Kaufman R J. Protein processing within the secretory pathway. Curr Opin Biotechnol 1992: 3: 560.

29. Nirunsuksiri W, Presland R B, Brumbaugh S G, Dale B A, Fleckman P. Decreased profilaggrin expression in ichthyosis vulgaris is a result of selectively impaired posttranscriptional control. J Biol Chem 1995: 270: 871.

30. Rand M D, Grimm L M, Artavanis-Tsakonas S et al. Calcium depletion dissociates and activates heterodimeric notch receptors. Mol Cell Biol 2000: 20: 1825.

31. Dale B A, Resing K A, Presland R B. Keratohyalin granule proteins. In: Leigh I, Lane B, Watt F, eds. The Keratinocyte Handbook. Cambridge.: Cambridge University Press, 1994: 323.

32. Haydock P V, Blomquist C, Brumbaugh S, Dale B A, Holbrook K A, Fleckman P. Antisense profilaggrin RNA delays and decreases profilaggrin expression and alters in vitro differentiation of rat epidermal keratinocytes. J Invest Dermatol 1993: 101: 118

33. Konda Y, Yokota H, Kayo $\mathrm{T}$ et al. Proprotein-processing endoprotease furin controls the growth and differentiation of gastric surface mucous cells. J Clin Invest 1997: 99: 1842.

34. Madison K C, Sando G N, Howard E J et al. Lamellar granule biogenesis: a role for ceramide glucosyltransferase, lysosomal enzyme transport, and the Golgi. J Investig Dermatol Symp Proc 1998: 3: 80.

35. Elias P M, Cullander C, Mauro $\mathrm{T}$ et al. The secretory granular cell: the outermost granular cell as a specialized secretory cell. J Investig Dermatol Symp Proc 1998: 3: 87.

36. Madison K C, Howard E J. Ceramides are transported through the Golgi apparatus in human keratinocytes in vitro. J Invest Dermatol 1996: 106: 1030.

37. Milner L A, Bigas A. Notch as a mediator of cell fate determination in hematopoiesis: evidence and speculation. Blood 1999: 93: 2431

38. Bigas A, Martin D I, Milner L A. Notch1 and Notch2 inhibit myeloid differentiation in response to different cytokines. Mol Cell Biol 1998: 18: 2324.

39. Milner L A, Bigas A, Kopan R, Brashem-Stein C, Bernstein I D, Martin D I. Inhibition of granulocytic differentiation by Notch1. Proc Natl Acad Sci U S A 1996: 93: 13014.

40. Perron M, Harris W A. Determination of vertebrate retinal progenitor cell fate by the Notch pathway and basic helixloop-helix transcription factors. Cell Mol Life Sci 2000: 57: 215

41. Rogister B, Ben-Hur T, Dubois-Dalcq M. From neural stem cells to myelinating oligodendrocytes. Mol Cell Neurosci 1999: 14: 287.

42. Favier B, Fliniaux I, Thelu J et al. Localisation of members of the notch system and the differentiation of vibrissa hair follicles: Receptors, ligands, and fringe modulators. Dev Dyn 2000: 218: 426.

43. Lin M H, Leimeister C, Gessler M, Kopan R. Activation of the Notch pathway in the hair cortex leads to aberrant differentiation of the adjacent hair-shaft layers. Development 2000: 127: 2421

44. Thelu J, Viallet J P, Dhouailly D. Differential expression pattern of the three Fringe genes is associated with epidermal differentiation. J Invest Dermatol 1998: 111: 903.

45. Kayo T, Sawada Y, Suda M et al. Proprotein-processing endoprotease furin controls growth of pancreatic beta-cells. Diabetes 1997: 46: 1296

46. Dale B A, Lonsdale-Eccles J D, Holbrook K A. Stratum corneum basic protein: an interfilamentous matrix protein of epidermal keratin. Curr Probl Dermatol 1980: 10: 311.

47. Ishida-Yamamoto A, Takahashi H, Presland R B, Dale B A, Iizuka $\mathrm{H}$. Translocation of profilaggrin N-terminal domain into keratinocyte nuclei with fragmented DNA in normal human skin and loricrin keratoderma. Lab Invest 1998: 78: 1245

48. Presland R B, Bassuk J A, Kimball J R, Dale B A. Characterization of two distinct calcium-binding sites in the amino-terminus of human profilaggrin. J Invest Dermatol 1995: 104: 218

49. Thulin C D, Walsh K A. Identification of the amino terminus of human filaggrin using differential LC/MS techniques: implications for profilaggrin processing. Biochemistry 1995: 34: 8687.

50. Presland R B, Haydock P V, Fleckman P, Nirunsuksiri W, Dale B A. Characterization of the human epidermal profilaggrin gene. Genomic organization and identification of an S-100-like calcium binding domain at the amino terminus. J Biol Chem 1992: 267: 23772

51. Markova N G, Marekov L N, Chipev C C, Gan S Q, Idler W W, Steinert P M. Profilaggrin is a major epidermal calcium-binding protein. Mol Cell Biol 1993: 13: 613.

52. Miyachi Y, Yoshimura N, Suzuki S et al. Biochemical demonstration and immunohistochemical localization of calpain in human skin. J Invest Dermatol 1986: 86: 346.

53. Suzuki Y, Nomura J, Koyama J, Horii I. The role of proteases in stratum corneum: involvement in stratum corneum desquamation. Arch Dermatol Res 1994: 286: 249. 